\title{
Design and Verification of Children-oriented Educational Application Based on Eye Movement Interactive Technology
}

\author{
Shu Wang ${ }^{1, a, *}$, Qing Wang ${ }^{1, b}$, and Hong Chen ${ }^{1, c}$ \\ ${ }^{1}$ College of Information and Electrical Engineering, China Agricultural University, Beijing 100083, \\ China \\ a460594932@qq.com, ${ }^{b}$ wangqingait@sina.com, ${ }^{c}$ norman_chen@263.net
}

Keywords: eye movement interaction, educational game, eye movement recognition, T-test.

\begin{abstract}
In recent years, games and educational products targeted to children have sprung up. However, the applications of most products are not suitable for children's current cognitive development level and operating habits, which may result in negative effects on children's attractiveness. In accordance to the specific behavior habits of children users, this paper has put forward a design principle of eye movement interactive system under educational game mode. At the same time, the application framework of children Educational-games Based Eye Interaction (EBEI) is constructed, as well. On this basis, an application of Insect Science Teaching Games based on eye movement interaction mode is designed. Moreover, T-test method is used to compare and analyze the practical application effects of the game. It shows in the experimental results that the game has strong usability and can better improve the enthusiasm of the children to study knowledge of insects and achieve good educational effects.
\end{abstract}

\section{Introduction}

With the development of science and technology, the types of games are constantly innovated. Children's love to the games encourages some educational games to come into being. The educational games oriented to children is a computer application software [1] that combines the purposes of specific teaching and entertainment. In a word, its essence is to teach in a form of playing games, so that the player can accept the expressions of knowledge in the state of entertainment, and then it achieves the purpose of interest and educational balance.

The human-machine interaction method used in traditional electronic games is mainly realized by external devices such as handles, keyboards or mouse. However, there are many different interactive modes of electronic game operation nowadays, which includes the technology of touch control, speech recognition, body interaction, gesture recognition, virtual reality and augmented reality, tactile feedbacks, EMG simulation, brain computer interaction, and so on. Due to the uniqueness of the 
designing purposes of educational games and the oriented objects, the ways of interaction have great limitations. The traditional human-computer interaction is widely used in the educational games in China, which is easy for children to have a sense of boring and fatigue when they are experiencing the game. At the same time, there is a phenomenon that children cannot operate the interactive equipment skillfully. All of these kinds of problems may shorten children's attention time to games. In addition, it not only weaken the playfulness, but also affect the learning effects. In view of the particularity of children users, that is, different from those of adults in the aspects of physiological characteristics, psychological characteristics and operation habits, children educational games should have the characteristics of relatively low interaction complexity and it also should have the abilities to maintain and improve the subjective initiative of children for a long time.

Based on the above analysis, this paper proposes the design principles of eye movement interactive application under the educational game mode based on the key technology of eye movement interaction while combining with the requirements of children's educational games. Furthermore, it also puts forward the application framework of children Educational-games Based Eye Interaction (EBEI), which is also used to design application of popular insect science teaching games for children's users. At the same time, T test method is used to compare and analyze the actual effects of the game. Finally, the experimental results show that the interactive way of eye movement can better improve the learning interests and learning effects of the players on insect knowledge, which has a great significance in science popularization and interests.

\section{Related Work}

Late-model human-computer interaction technology is moving towards the goal of human-centered, natural and high efficient development, so that users can use a variety of sensory channels for interaction[2]. Among them, eye movement is a more natural and latest mode for human-machine interaction. The interaction of eye movement depends on the technology of tracking the sight line. The early methods of tracking the sight line mainly include observation and mechanical recording. However, in the last one hundred years, the methods of tracking the sight line has begun to appear on the objective observation and recording of eye movement based on hardware devices[3]. Among the methods of eye tracking, optical recording method has the longest research history and it has the most research achievements. Therefore, it is the most important application type of modern eye tracking instrument[4]. With the development of the miniaturization of eye tracking equipment, many research teams at home and abroad have developed a series of eye movement interactive prototype systems, such as the eye controlled wheelchair system developed by the Barea team in 2002 [5]. In 2006, Ward and others developed a simple eye movement text input system [6]. In 2006, David and others in Canada has first applied eye movement to video games [7]. In 2008, Marco Porta and others realized through input control through eye series, and developed a Eye-S system which could provide general eye movement input [8]. In the same year, Tomasz and others proposed an application controlled by using blink manipulation [9]. In China, in 2011, Pan Gang, professor in Zhejiang University and others have developed an eye-movement interactive painting system, which is faced to people with serious limb defects [10]. In 2012, Li Ting, professor in Zhejiang University have applied the eye-movement interaction system into a vehicle-mounted head-up display (HUD) and developed the Eye HUD, which is a case of an eye-assisted interactive system [11]. In 2014, song Han Xiao and others put forward an eye movement password input method based on blinking and staring input [12].

The interactive mode based on eye movement has become a hot spot in recent researches. However, it has not been involved in the field of educational games. Most of the educational games has adopted the traditional interactive ways, such as keyboards and mouse. In recent years, part of educational 
games have adopted a new way of interaction. For example, the educational game was developed based on the physical interaction, which can not only activate the interests of active learning but also extend the content of the learning from the text to the skill of physical activities, so that the players will no longer be stuck in front of the computers. However, for the reason that there are many problems of precision and delay in somatosensory equipment, the recognition ability of many subtle action is poor, and the recognition of complex action is fuzzy, as well as the time delay. Therefore, it results in the effects on the transmission of educational content[13]. The real educational games are strengthened by scanning the real things through mobile phones or tablets, and then superimpose their virtual education content into the real scene, so as to enhance the sense of authenticity and immersion of knowledge acquisition and provide a new way of thinking for children's education. But for younger children, there is a possibility of not being able to manipulate interactive devices alone [14].

Based on the above analysis, the interaction form controlled by eye movement provides a more natural interaction experience. In the cognitive process, vision and hearing is the starting point to understand the world. In addition, human beings get outside information through the ears and eyes, and the motion instructions will be formed through the cognitive processor, so as to guide other interactive forms, such as clicking the mouse, touching the screen and so on, as is shown in Figure 1. Therefore, eye movement interaction is more suitable for the children with lower cognitive ability. In addition, the fine muscles of the eye movement have good immunity to fatigue. In the long time of interactive experience, it can ensure the accuracy and maneuverability, as well as giving better interactive experience to the children.

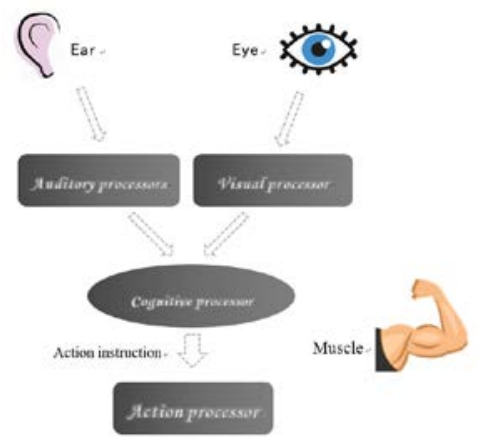

Fig. 1 Human cognitive process

\section{The Interactive Design of Eye Movement for Children's Educational Games}

Based on the characteristics of educational games and the interactive mode of eye movement, this paper proposes the design principles of eye movement interactive system for children's educational games, which mainly include the following four aspects:

(1) Graphical user interface: In accordance to children users, the characteristics like educational level and cognitive development are significantly different from adults, so the interactive interface should be more targeted. In the process of designing Educational-games Based Eye Interaction for children, the user interface should be graphical and symbolic, so as to reduce the use of words and the burden of children's memory.

(2) Simple eye interaction set: In view of the effective transmission of educational content, the learning time of interaction should be reduced. Therefore, the types of eye movements should be simplified. At the same time, eye interaction sets should be defined conformed to the cognitive level of children, so as to simplify the input mechanism. For example, it can be defined like gaze, blinking eyes and other eye interactions which conform to the visual attention mechanism of children. On the 
contrary, a single eye blink or eye dragging and other interactions which requires high accuracy and difficult operation, should be avoided. Thus, eye interaction can be used as a simple and natural interaction channel in educational games.

(3) Multi-channel cooperation: In the view of children, complex eye movement interaction cannot map all interactive commands while they can better complete interactive tasks if taking the use of other channels for auxiliary eye movement, such as keyboard, gesture, and so on. In addition, it can also improve efficiency and accuracy of the interaction, as well as effectively reducing the problems caused by Midas Touch.

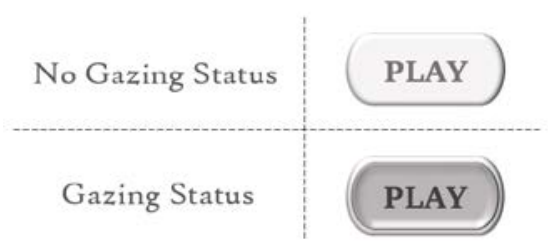

Fig. 2 Visual feedback

(4) Visual feedbacks: Visual feedback includes two parts: eye movement auxiliary feedbacks and content feedbacks. Eye movement aided feedbacks can effectively improve the accuracy of interaction and help alleviate visual fatigue. For example, when a button is gazed at, the current gazing action is recognized by the state changes of the button, as is shown in Figure 2. Content feedback is part of the transmission of educational content. Educational games should be driven by knowledge points, and make appropriate visual feedback in the course of the game, so that players will transform from passive learning into active learning state, and then it can guide players to have reflective observation and exploration.

\subsection{EBEI framework}

Based on the above interaction principles, this paper proposed an application framework for EBEI (Educational-games Based Eye Interaction), as is shown in Figure 3.

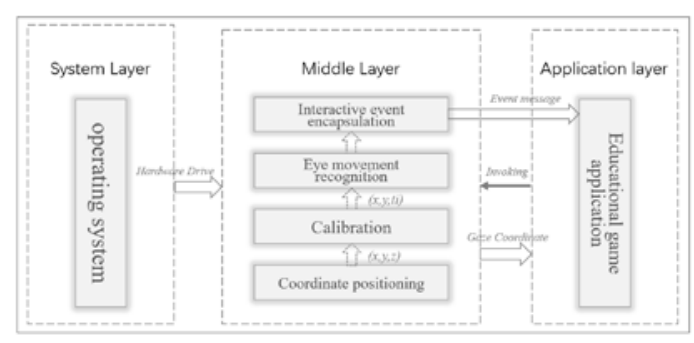

Fig. 3 EBEI framework

This application framework is from the bottom to the up, which includes the system layer, the middle layer and the application layer. At the bottom, the system layer mainly provide the camera and infrared devices by hardware and the corresponding parameters are set. The middle layer is mainly composed of calibration module of eye location module, eye action recognition module and other interactive event encapsulation modules. Firstly, the user's eye space coordinate system is established in the line of sight positioning module, and the user's gazing direction $\mathrm{p}(\mathrm{x}, \mathrm{y}, \mathrm{z})$ in the space coordinate system will be obtained in real time. Secondly, in the calibration module, it will be converted to the $\mathrm{g}(\mathrm{x}, \mathrm{y}, \mathrm{ti})$ in the screen scene coordinate system according to the mapping relationship, in which $\mathrm{X}$ and $\mathrm{y}$ are the user's fixation points on the screen scene. ti represents the time point of the system. Thirdly, in the eye movement recognition module, the user eye interactive action set is defined by combining 
with the corresponding eye movement input mechanism, so as to determine whether the current existence and what type of eye movements exist according to a certain context. Finally, in the interactive event encapsulation module, eye movement assistance event mapping is generated and encapsulated by combining other channel input commands such as keyboard and gesture. In the application layer, the real-time eye movement coordinate and the event message can be called is encapsulated, so as to replace the mouse movement and activate the corresponding interactive function. Therefore, the interactive operation and control of the educational game application can be realized.

\subsection{Recognition of eye movement interaction}

According to the design principles proposed in this paper, three types of eye interaction actions are constructed, as is shown in table 1 . The gazing movement is to keep eyes in the target area, and the double blink of the eye requires two consecutive blinks in a short time, aimed to distinguish the normal blink of the eyes. The saccade behavior refers to the rapid jump between the two or more target areas.

Table 1 Types of eye interaction actions

\begin{tabular}{ll}
\hline Eye interaction & Description \\
\hline fixation & Stare in the target area \\
blink-2 & Two consecutive blinks \\
saccade-2 & Saccade in the target area \\
\hline
\end{tabular}

Considering the weakness of children's interaction ability, we only define the saccade action in two target areas. For example, within a customized two-point saccade threshold, the gaze point ahould be implemented by the $\mathrm{a} 0$ (the conventional close button position) in the upper right corner of the interface. The exit command can be implemented through any path pn to the upper left corner of the interface, as is shown in Figure 4.

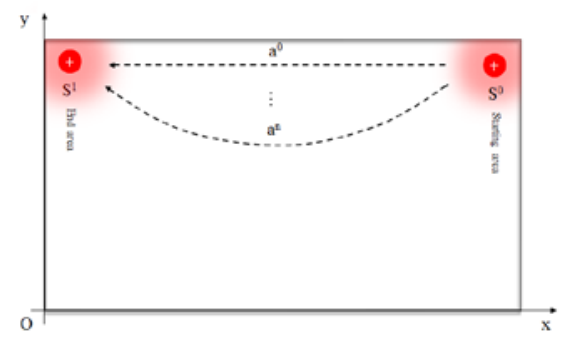

Fig. 4 Exit instructions for two gaze point saccades

The recognition process of two point saccade action shown in Figure 4 is in the time period $<t_{i}, t_{j}>$. The moment $t_{i}$ is viewed as the time point coordinates of $P_{i}\left(x_{i}, y_{i}, t_{i}\right)$, and the moment $t_{j}$ is viewed as the point coordinates of $P_{j}\left(x_{j}, y_{j}, t_{j}\right)$. At the same time, $\mathrm{S}_{1}$, and $\mathrm{S}_{2}$ is defined as the target areas by the system.

$$
\mathrm{S}_{1}=\left\{\begin{array}{l}
x_{1} \in\left[x_{\min 1}, x_{\max 1}\right] \\
y_{1} \in\left[y_{\min 1}, y_{\max 1}\right]
\end{array} \quad \text { (1) } \quad \mathrm{S}_{2}=\left\{\begin{array}{l}
x_{2} \in\left[x_{\min 2}, x_{\max 2}\right] \\
y_{2} \in\left[y_{\min 2}, y_{\max 2}\right]
\end{array}\right.\right.
$$

If the conditions (3) and (4) are satisfied, the gaze point has changed from the target area $S_{1}$ to the target area $S_{2}$ within the predefined time threshold. Finally, the eye-hopping action will be 
successfully recognized.

$$
\exists t_{j}-t_{i} \leq \varepsilon_{T}
$$

$$
\left\{\begin{array} { l } 
{ x _ { i } \in [ x _ { \operatorname { m i n } 1 } , x _ { \operatorname { m a x } 1 } ] } \\
{ y _ { i } \in [ y _ { \operatorname { m i n } 1 } , y _ { \operatorname { m a x } 1 } ] }
\end{array} \wedge \left\{\begin{array}{l}
x_{j} \in\left[x_{\min 2}, x_{\max 2}\right] \\
y_{j} \in\left[y_{\min 2}, y_{\max 2}\right]
\end{array}\right.\right.
$$

In the equation, $\varepsilon_{T}$ is a predefined saccade time threshold.

\section{System Design}

Based on the framework of EBEI and using the Unity3D cross platform development engine as a development tool, this paper designs and implements an educational game application of eye movement interactive insect recognition. In the game, children can identify insect species through controlling eye movements and learn related entomological knowledge, so as to achieve the purpose of combining interest with learning. The game chooses the design that combines the eye movement interactive technology with the educational game mode. At the same time, the control mode of the game will be simplified, and the fun and maneuverability of the game better can be improved. In addition, it also opens new development ideas for the field of educational games.

\subsection{Overall design}

The game level is set in different scenes, which can better map the daily living environment. When the game starts, the caves distributed in the game scene are randomly assigned to an insect at a set intervals. The player locks or releases the insect through the eye movements. If it is a pest, the player can beat the insect by blinking the eyes. If the player eliminates the pests correctly, the score will be gained. In the other hand, if eliminating the beneficial insects, the corresponding scores will be cut down. With the increase of the number of checkpoints, insects will appear faster and faster, and interference items (beneficial insect) will become more and more. At the same time, the game will give feedback in the form of corresponding visualization, mainly for insect science knowledge. After the game, the screen will show the final score, accuracy and response speed. The system flow is shown in Figure 5.



Fig. 5 System flow chart 


\subsection{Interactive design}

The game consists of four parts: calibration, menu selection, game player, feedbacks and score accounting. The interface is shown in Figure 6.

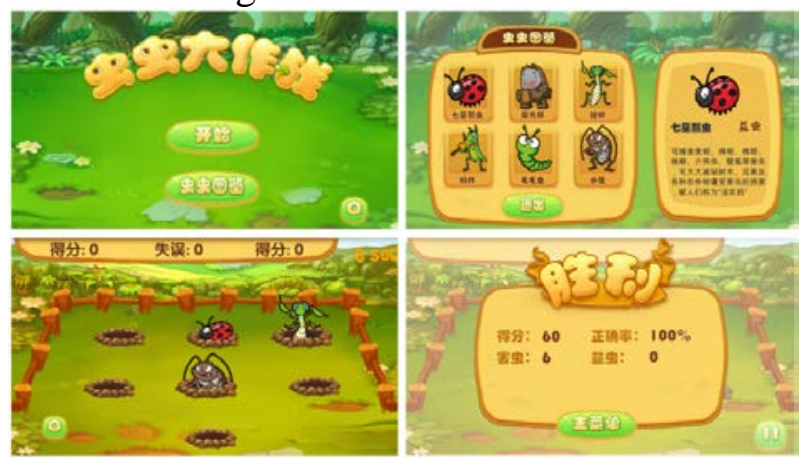

Fig. 6 Game Interface

At the end of the calibration, children are prompted by the interface to move their gaze at will and complete the corresponding operation. The interactive instructions corresponding to eye movements in this system are shown in table 2. Players can lock up an option by staring action, and confirm the lock option for two consecutive blinks. You can exit or interrupt the game at any time during the game, and jump into the scene of the pictorial view.

Table 2 Eye movement instruction table

\begin{tabular}{ll}
\hline Eye Movement & Instructions \\
\hline Gazing & Lock or release \\
$\begin{array}{l}\text { Two consecutive blinks } \\
\text { Line of sight moving from the upper right to the upper left }\end{array}$ & Confirm \\
corner & Exit or return \\
$\begin{array}{ll}\text { Line of sight moving from the lower left corner to the upper } \\
\text { left corner }\end{array}$ & $\begin{array}{l}\text { Interrupt the game to check the } \\
\text { pictorial view }\end{array}$ \\
\hline
\end{tabular}

\section{Experimental Verification}

In accordance to the educational game based on eye movement interaction, it requires to be verified in three aspects. Firstly, the usability of the game should be evaluated; Secondly, the playability of the game should be evaluated; Thirdly, the educational accessibility of the game should be evaluated, as well. For this purpose, 28 preschool children aged from four to six were selected for the evaluation of usability, playability and educational accessibility.

\subsection{Experiment for usability}

Usability evaluation is directly related to the quality of the game. The usability of the game is whether the player can effectively understand and control the game autonomously [15]. In the application of this game, usability is mainly reflected in the player's adaptation to eye movement interaction and understanding of game play. In order to verify the usability of the game, 10 children were randomly selected from 28 children to play independently without the guidance of the relevant personnel. The results showed that only three children were able to ask for help, while the rest were able to play the game on their own until the end of the game. At the end of the experiment, all children were asked how they felt about this interaction mode. No one felt dizzy, which can confirm that the 
interaction mode is available and operable.

\subsection{Experiment for playability}

The playability of the game is the attraction of the game itself to the players, and it is the vitality of the game, as well. In educational games, the lack or excessive playability of the game will lead to the unsatisfactory effects of knowledge transmission, which may greatly influenced the educational accessibility of the game. In order to verify the playability and entertainment effects of the game, we choose a mouse interaction game based on keyboard interaction as a contrast. Twenty-eight children were randomly divided into two groups. The comparable group C-G was assigned to play a hamster game based on keyboard interaction. The experimental group E-G played the game designed in this paper. Valid recorded data are included the time Dt the player volunteered to play, the interval Rt between restarts after the game failed, and the number Lr of levels the player passed. The experiment does not have the time limit. Therefore, from the comparative data, the degree of player's enthusiasm for the game can be seen, thereby verifying the game's playability. The recorded data is shown in Table 3.

Table 3 Playability test record (min)

\begin{tabular}{cccc|lcccc}
\hline ID & Dt & Rt & Lr & ID & Dt & Rt & Lr \\
\hline 1 & 6 & 6 & 1 & 15 & 8 & 5 & 3 \\
2 & 7 & 5 & 3 & 16 & 10 & 3 & 3 \\
3 & 10 & 8 & 2 & 17 & 8 & 6 & 3 \\
4 & 6 & 5 & 1 & 18 & 9 & 3 & 3 \\
5 & 9 & 4 & 2 & 19 & 11 & 5 & 2 \\
6 & 11 & 3 & 3 & 20 & 7 & 4 & 3 \\
C-G & 8 & 5 & 3 & E-G & 21 & 9 & 5 & 2 \\
8 & 10 & 3 & 3 & 22 & 8 & 5 & 3 \\
9 & 10 & 4 & 3 & 23 & 10 & 4 & 1 \\
10 & 8 & 0 & 3 & 24 & 8 & 3 & 3 \\
11 & 7 & 7 & 2 & 25 & 10 & 3 & 3 \\
12 & 13 & 8 & 1 & 26 & 6 & 5 & 2 \\
13 & 6 & 4 & 2 & 27 & 8 & 3 & 3 \\
14 & 10 & 5 & 3 & 28 & 8 & 5 & 3 \\
\hline
\end{tabular}

For the reason that the sample size is small, this paper determined the use of T-Test to judge whether there is a significant difference between the two groups of data. Therefore, the two groups of data were compared and analyzed. This experiment selects 0.05 as a significant level, so as to calculate the data $\mathrm{P}$ in Table 3, as is shown in Table 4.

Table $4 \mathrm{P}$ value of Playability T test

\begin{tabular}{ccc}
\hline $\mathrm{Dt}$ & $\mathrm{Rt}$ & $\mathrm{Lr}$ \\
\hline 0.916396 & 0.374275 & 0.21032 \\
\hline
\end{tabular}

It is showed that for the data in duration Dt (min), $P=0.916396(>0.05)$ in the results, which meant that there was no significant difference in the duration of the game between the two groups. In accordance to the retest times Rt (min), $\mathrm{P}=0.374275(>0.05)$, which means that the degree of game motivation is at the same level. In accordance to the number $\mathrm{Lr}$ of all the levels that children have passed, it indicates that the difficulty of the two matches is almost the same. Through the analysis of 
the above data, children have no difference in the experience of the two games. The application of this game based on eye movement is equally attractive to the traditional ones, thus verifying the playability of the game.

\subsection{Experiment for educational accessibility}

The accessibility of education is the key to the difference between educational games and general games [16]. Whether players can understand knowledge of related disciplines in games is the value criterion of educational games. In order to compare the differences between the ability of knowledge popularization of this game and the effects of traditional education, 28 children were randomly divided into two groups to learn different ways: the experiment group learned the insect related knowledge through this game, and the comparable group learned the insect related knowledge through the given insect map. Before the experiment, the subjects were arranged to conduct a questionnaire survey, which is aimed to test insect related knowledge background. The questionnaire includes ten kinds of insects. It is necessary to write the corresponding name according to the appearance of each insect and determine the insect pest attribute. The number of correct entries was recorded, aimed to determine their prior knowledge level. Therefore, it can be compared with the results after testing. After the experiment, the subjects were asked to conduct the same questionnaire. The results are shown in table 5 and table 6.

Table 5 Educational accessibility test record (min)

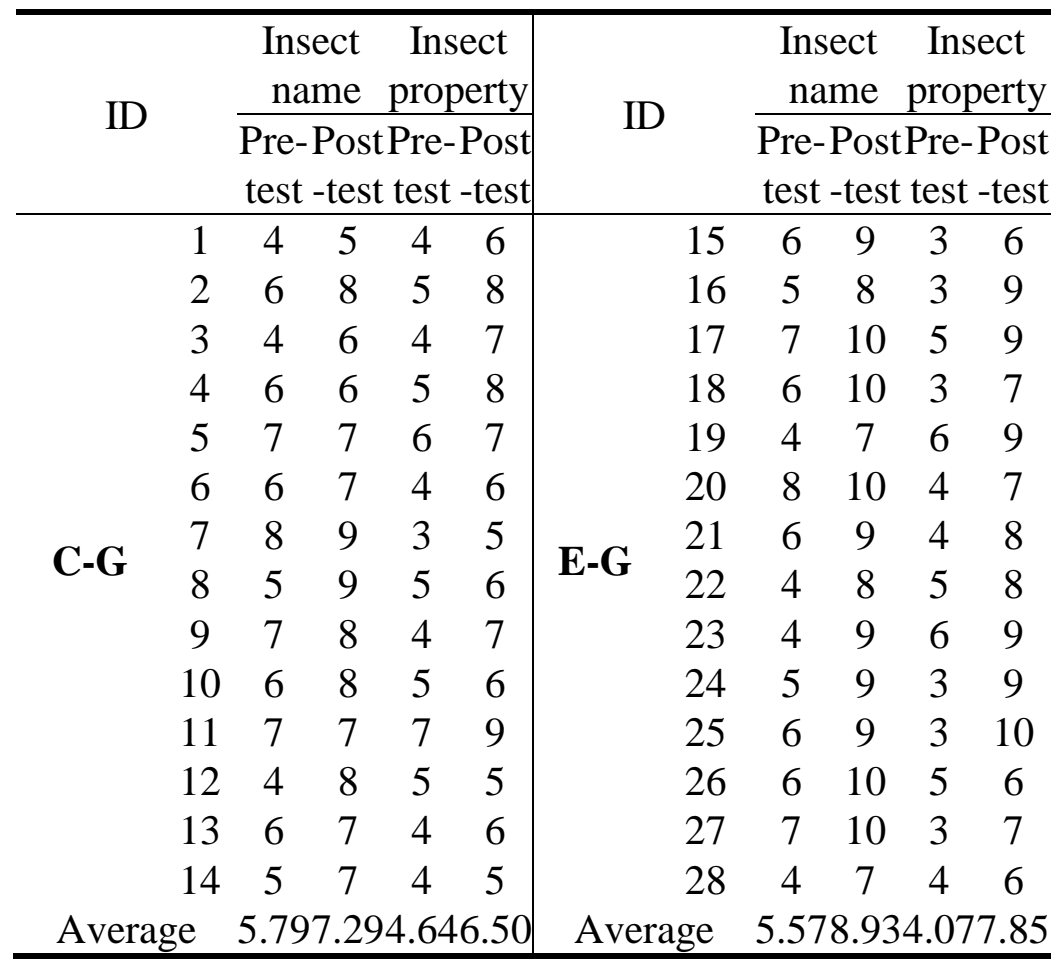

Table $6 \mathrm{P}$ value of Educational accessibility $\mathrm{T}$ test

\begin{tabular}{cc|cc}
\hline \multicolumn{2}{c|}{ Insect name } & \multicolumn{2}{c}{ Insect } \\
\hline Pre-test & Post-test & Pre-test & Post-test \\
\hline 0.658424 & 0.000559 & 0.60593 & 0.009849 \\
\hline
\end{tabular}

In table 6 the values of P respectively were 0.658482 and 0.60593 in T-test of the insect names of the prior knowledge level and the insect attributes, which were all more than 0.05 . It is meant that two 
samples had the same knowledge level in these contents. After the experiment, the two groups all have improved the results of the same questionnaire, which showed that the educational application of the eye movement interaction was communicated with the traditional methods. However, after the comparative analysis, the test value $\mathrm{P}$ of the two groups respectively were 0.000559 and 0.009849 (all less than 0.05). There was a significant difference between the two groups in the results. The average score of the experimental group was higher than that of the comparable group, which suggests that this educational game based on eye movement interaction could produce better educational effects.

\section{Conclusions}

In this paper, it puts forward the design principles of eye movement interactive application under the educational game mode based on the key technology of eye movement interaction while combining with the characteristics of children's behaviors. Furthermore, it also puts forward the application framework of children Educational-games Based Eye Interaction (EBEI), based on the unique behavior habits of children group. It also defines eye interactive action set, and designs an application of popular science teaching games for children's users. At last, the T-TEST is applied to analyze the difference of the game application. The analysis results show that the game has strong usability, which can better improve children's enthusiasm for insect knowledge learning and achieve good educational results.

\section{Acknowledgments}

This work was financially supported by National Key Technology R\&D Program of the Ministry of Science and Technology fund (Program number: 2015BAK04B00).

\section{References}

[1] Shen Juan, Zhang Sujing. A Review of Educational Games Assessment at Home and Abroad [J]. Journal of Distance Education, 2014, 32(03):105-112.

[2] Shuiqiang Lin. The Research and Application of Natural Human-Computer Interaction Key Technology [D]. Mianyang: Southwest University of Science and Technology, 2015.

[3] Juan Xu. Development and performance comparison about eye trackers [J]. China Modern Educational Equipment, 2012, (23):16-18.

[4] Yuchang Han. The Developmental History of the Eye Movement Apparatus and the Experimental Method of Eye Movement [J]. PSYCHOLOGICAL SCIENCE, 2000,(04):454-457.

[5] Barea R, Boquete L, Mazo M, et al. System for assisted mobility using eye movements based on electrooculography[J]. IEEE Transactions on Neural Systems \& Rehabilitation Engineering, 2002, 10(4):209-218.

[6] S.A. Wills,D.J.C. MacKay. DASHER-an efficient writing system for brain-computer interfaces? [J]. IEEE Transactions on Neural Systems and Rehabilitation Engineering, 2002, 14(2):244-246.

[7] Smith J D, Graham T C N. Use of eye movements for video game control: International Conference on Advances in Computer Entertainment Technology, 2006 [C]. New York: ACM, 2006.

[8] Marco Porta and Matteo Turnia. Eye-S: A Full-Screen Input Modality for Pure Eye based Communication: ETRA, 2008 [C]. Savannah, Georgia, 2008.

[9] Tomasz Pander, Tomasz Przybyla, and Robert Czabanski. An Application of Detection Function for the Eye Blinking Detection: HIS,2008 [C].Krakow, Poland, 2008.

[10] LI Shan, PAN Gang, LI Shi-jian. Eye-Controlled Painting System for Disabled [J]. ACTA ELECTRONICA SINICA, 2011 (1): 163-167

[11] Ting Li. Eye movement interactive interface design and case development [D].Hangzhou:Zhejiang University,2012. [12] Xiaohan Song, Wei Hu, Guihuan Feng. An Eye and Keyboard Integrated Password Input Method: HHME2014[C]. Beijing. 2014: 1-7.

[13] Qing Li, Qing Wang. Motion Sensing Technology in Education [J]. Journal of Distance Education, 2015, 33(01):48-56. 
[14] Lifang Xu, Cheng Chi, Qi Zhang. On the Research about Educational Games Based on Augmented Reality Technology [J]. Journal of Xiangtan University (Philosophy and Social Sciences), 2015, 39(02):120-123.

[15] David, Pinelle, Nelson, Wong, Tadeusz, Stach. Heuristic Evaluation for Games: Usability Principles for Video Game Design[C]. Florence.2008:1453-1462.

[16] Senlin Lv. Educational Game Industry Research Report [J]. Distance Education in China, 2004, (22): 44-47 